Sergio Oswaldo Zhingre Orellana; Juan Carlos Erazo Álvarez; Cecilia Ivonne Narváez Zurita; Verónica Paulina Moreno

http://dx.doi.org/10.35381/r.k.v5i10.699

\title{
Plan de negocios para evaluar la viabilidad en el sector microempresarial de repuestos de vehículos
}

\section{Business plan to assess the viability in the micro-business sector of vehicle spare parts}

Sergio Oswaldo Zhingre-Orellana

sergio.zhingre@psg.ucacue.edu.ec

Universidad Católica de Cuenca, Cuenca

Ecuador

https://orcid.org/0000-0002-3442-6996

Juan Carlos Erazo-Álvarez

jcerazo@ucacue.edu.ec

Universidad Católica de Cuenca, Cuenca

Ecuador

https://orcid.org/0000-0001-6480-2270

Cecilia Ivonne Narváez-Zurita

inarvaez@ucacue.edu.ec

Universidad Católica de Cuenca, Cuenca

Ecuador

https://orcid.org/0000-0002-7437-9880

Verónica Paulina Moreno

verónica.moreno@ucacue.edu.ec

Universidad Católica de Cuenca, Cuenca

Ecuador

https://orcid.org/0000-0003-1517-6124

Recibido: 20 de marzo de 2020

Revisado: 05 de abril de 2020

Aprobado: 20 de abril de 2020

Publicado: 19 de mayo de 2020 


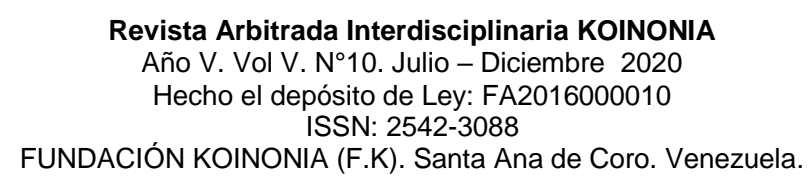

Sergio Oswaldo Zhingre Orellana; Juan Carlos Erazo Álvarez; Cecilia Ivonne Narváez Zurita; Verónica Paulina Moreno

\title{
RESUMEN
}

El objetivo de la investigación fue la creación de un plan de negocio para comercializar repuestos para el parque automotor del cantón Gualaceo. La metodología que se aplicó en este estudio es una investigación descriptiva con un enfoque mixto y aplicando el método deductivo-inductivo. Como resultado de esta investigación se determinó a los principales competidores, tipo de vehículos que existen en el cantón y la poca satisfacción de los consumidores, realizando un esquema que al analizar sus factores nos dan resultados positivos y que permite visualizar, aprovechar la oportunidad de negocio. El plan de negocios es una herramienta de gran importancia pues permite planificar la creación de empresas con el propósito de desarrollar el negocio de la forma más eficiente posible.

Descriptores: Empresa; estudio de mercado; estudio de audiencia; vehículo automotor. (Palabras tomadas del Tesauro UNESCO).

\begin{abstract}
The objective of this research was to create a business plan to market spare parts for the automotive park in the Gualaceo canton. The methodology applied in this study was a descriptive investigation with a mixed-methods approach in addition to the deductiveinductive method. As a result of this research, the main competitors, type of vehicles that exist in the canton and the low satisfaction of consumers were determined, making a scheme that, when analyzing their factors, gives us positive results and allows us to visualize and take advantage of the business opportunity. The business plan is a tool of great importance since it allows planning the creation of companies with the purpose of developing the business in the most efficient possible way.
\end{abstract}

Descriptors: Enterprises; market research; audience research; motor vehicles. (Words taken from the UNESCO Thesaurus).

\section{INTRODUCCIÓN}

En la última década el desarrollo automotriz en el Ecuador, se ha convertido en un aporte importante para la economía nacional, por los ingresos que genera, por su fabricación y los impuestos generados al fisco. La Asociación de Empresas Automotrices del Ecuador (2018) afirma. "Su dinamización contribuyó con USD 1.633 millones en el 2018 en tributos al Estado, es decir, USD 738 millones más que el 2016" 


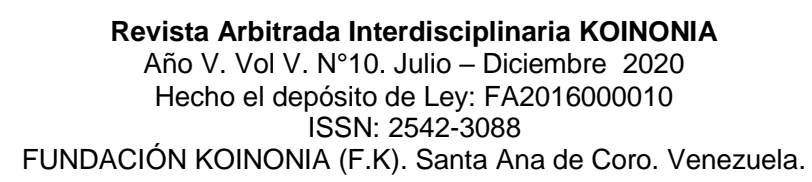

Sergio Oswaldo Zhingre Orellana; Juan Carlos Erazo Álvarez; Cecilia Ivonne Narváez Zurita; Verónica Paulina Moreno

(p. 40). También su mano de obra directa e indirecta y las numerosas plazas de trabajo y empleos que ofrece este sector. "se afiliaron 3.791 nuevos trabajadores en las empresas de producción, comercialización y mantenimiento de vehículos motorizados, este incremento significativo representa un crecimiento del $5.9 \%$ y hasta el momento el sector registra 68.155 afiliados" (Asociación de Empresas Automotrices del Ecuador, 2018, p.40).

En el 2015, se matricularon 1'925.368 vehículos motorizados en Ecuador, 57\% más que lo registrado en el 2010 cuando la cifra llegó a 1'226.349, según los últimos datos del Anuario de Transportes 2015, publicado por el Instituto Nacional de Estadística y Censos (Instituto Nacional de Estadisticas y Censos, INEC, 2016).

El crecimiento automotriz se debe a la estabilidad económica del país, facilidades de pago y la eliminación restricciones para los vehículos nuevos realizando un gran aporte la entrada de nuevas marcas y modelos. "El parque automotor matriculado en Ecuador creció en más de 1,4 millones de vehículos en una década, lo que situó la cifra por sobre los 2,4 millones de unidades a 2018" (El Comercio, 2019). Actualmente, en la provincia del Azuay la demanda de partes y repuestos automotrices está sobrepasando la oferta del sector comercial al alto crecimiento del parque automotor, y este estudio contribuirá a la factibilidad de apertura de una comercializadora de repuestos analizando su aspecto económico y financiero, generando directa e indirectamente trabajo y empleo que es un estímulo a la economía local.

Todos los días los vehículos transitan dentro y fuera de la cuidad encontrándose con algún tipo de accidente de tránsito lo cual son choques, volques, colisiones siniestros, estropeando así los vehículos a tal manera que el almacén de partes y repuestos son las beneficiaras directas de esta dura y triste situación. En el cantón Gualaceo la escases de proyectos de factibilidad para este sector automotriz ya sea por falta de conocimiento o interés por parte de propietarios de algunos almacenes que ya están en operación que no se dedican exclusivamente a la comercialización de repuestos, para las carrocerías de los vehículos no presentan una variedad y disponibilidad inmediata para el cliente. 
Sergio Oswaldo Zhingre Orellana; Juan Carlos Erazo Álvarez; Cecilia Ivonne Narváez Zurita; Verónica Paulina Moreno

Esto hace que genere molestias, por eso se ve la necesidad de la implementación de un plan de negocios para la comercializadora de partes y repuestos que se dedique exclusivamente a la venta de partes y repuestos automotrices en el cantón, es así, exigiendo y obligando a los dueños de los vehículos a trasladarse a otras ciudades para la adquisición de los mismos, causando a las personas pérdida de tiempo, paralización en su trabajo, aumento de gastos y la inseguridad de conseguir o no el repuesto necesario.

\section{Referencial teórico}

\section{Los estudios a nivel de factibilidad}

El término factibilidad es la posibilidad que tiene a realizarse o no ciertos objetivos 0 proyectos planteados, es un análisis que, en el caso de las empresas, se realiza con anterioridad para determinar si el negocio que se propone será bueno o malo y cuál es el camino adecuado para alcanzar el éxito. Una presentación de factibilidad es un informe de alta gestión. Este documento no demuestra de manera anticipada y concisa si el proyecto a ejecutarse puede continuar o de otra manera eficaz tratar de buscar otro camino adecuado para la resolución de un problema identificado. Para realizar estas estimaciones de factibilidad hay que considerar algunos criterios (como fuerza de capital, técnicos y otros), y algunas alternativas. Este análisis en su etapa inicial es muy importante realizarlo lo cual proyectara el desarrollo del proyecto (Peña-Abreu, Rodríguez-Rodríguez \& Piñero-Pérez, 2017).

Este estudio de factibilidad tiene el objetivo de demostrar la factibilidad económica y financiera para comercializar repuestos para el parque automotor del cantón Gualaceo. Dubs-de-Moya (2002) afirma. "que consiste en la investigación, elaboración y desarrollo de una propuesta de un modelo operativo viable para solucionar problemas, requerimientos o necesidades de organizaciones o grupos sociales" (p. 6). Al realizar el estudio de factibilidad es crear una propuesta de acción encaminada a resolver los problemas de las necesidades sociales o empresariales en general. 
Asimismo, Burneo-Valarezo, Delgado-Víctore \& Vérez (2016), sostienen que "La factibilidad de los proyectos en el sistema de dirección y gestión, con el propósito de establecer una secuencia de ejecución de los proyectos de inversiones, atendiendo a las prioridades en función de los indicadores que brindan los estudios" (p.305). Para poder aceptar la factibilidad se solicita un análisis eficaz y una precisa estimación de los indicadores financieros de la empresa, que nos señala si es factible o no la apertura de una comercializadora de repuestos (Puente \& Carrasco, 2017).

La elaboración de un estudio de factibilidad es muy importante para la toma de decisiones de los directores, administradores los cuales tienen la dura responsabilidad en cuanto dar pase o decretar las inversiones a realizarse basándose en los indicadores calculados, como el período de recuperación (PR), el Valor Actual Neto (VAN) y Tasa de Retorno (TIR), con estos indicadores se procede a elegir estrategias que guíen la ejecución y desarrollo del proyecto en base a los indicadores obtenidos y recursos disponibles (Burneo-Valarezo, Delgado-Víctore \& Vérez, 2016).

Las pequeñas y medianas empresas, llamadas también PYMES, han sido de gran importancia y contribución para la economía del país, así mejorando la economía de sus habitantes e ir ganando mercado a las grandes empresas y generando empleo local. El estudio de factibilidad tiene diferentes características que se debe de tener en cuenta al momento de su análisis como es la recuperación del capital o inversión a largo plazo, de tal manera que comprometen los recursos de la empresa y en sacrificios del personal, este es una inversión que se aplica a los activos o a aquellas acciones que demuestren el crecimiento de la empresa, persona o mercado (Esparza, Donoso, Esparza, Parada, \& Janeta, 2017).

Para el efecto se necesitan realizar varios estudios para justificar la viabilidad de la inversión, recuperación de capital invertido o entender las ventajas personales o grupales. Sin embargo, la información debe de ser precisa y clara para el rechazo o aceptación de la inversión y a la toma de decisiones apegada a la realidad, es necesario tener la información convincente para dar posibles soluciones, se debe de tener las características de los productos o servicios, maquinas, tecnologías a utilizarse. 


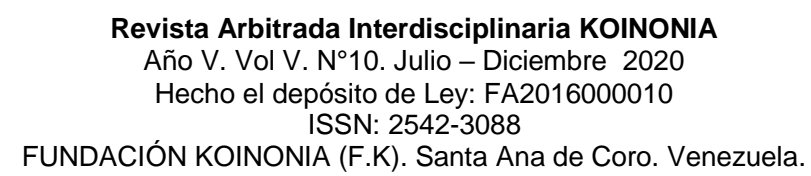

Sergio Oswaldo Zhingre Orellana; Juan Carlos Erazo Álvarez; Cecilia Ivonne Narváez Zurita; Verónica Paulina Moreno

Según (Esparza, Donoso, Esparza, Parada, \& Janeta, 2017) la estructura de una factibiliad esta compuesta por los siguentes elementos:

1. La investigación de mercado

2. El estudio técnico y de ingeniería

3. El estudio financiero

4. El estudio administrativo organizacional

5. El estudio de impacto ambiental

6. La evaluación del proyecto

A continuación se puntualiza los elementos que contiene un estudio de factibilidad.

\section{Estudio de mercado}

Es una herramienta que recolecta información sobre las necesidades de los consumidores, de esta manera se realiza un seguimiento y se investiga su comportamiento, analizando sus experiencias, características y determinando las principales causas o motivos de compra, así como las influencias ambientales, culturales políticas u otras causas al momento de compra, en esta fase es importante generar los productos que el mercado desee, es decir, establecer la viabilidad técnica de los productos o servicios (Argote, Vargas \& Villada, 2013).

\section{Estudio técnico y de ingeniería}

Para realizar un estudio técnico se debe de realizar un análisis general de la compania pero relacionando con los elementos especificos del proyecto, que esta a su vez nos lleve a la dificultad asolucionar, realizando un arbol de problemas al inicio del estudio de factibilidad, sus causas y efecto. Analizando los elementos anteriores nos permitira precisar el problema para realizar este análisis se debera contar con la area técnica o la ingenieria basica para determinar la precisión del proyecto. Para realizar este estudio se relaciona con la demanda estimada para poder adecuar las instalaciones de operación teniendo en cuenta la produccion, venta y distribución de los productos (PadillaCampoverde, Erazo-Álvarez \& Narváez-Zurita, 2019). 
Sergio Oswaldo Zhingre Orellana; Juan Carlos Erazo Álvarez; Cecilia Ivonne Narváez Zurita; Verónica Paulina Moreno

\section{Estudio administrativo}

Es una tarea en donde el administrados pone en acción los objetivos propuestos atravéz de la planeación, organización ,ejeucion, direccion y control para alcanzar los objetivos de la manera mas adecuada, es la aplicación de posibles soluciones a problemas determinadas (Marín \& Atencio, 2008). Asi mismo en este estudio se establece el funcionamiento organizacional, por otra parte, se realiza la constitución jurídica de ella, es decir, los trámites legales que debe cumplir para su funcionamiento dentro del marco legal (Murillo-Vilela, Erazo-Álvarez, Quevedo-Vázquez \& NarváezZurita, 2019).

\section{Estudio financiero}

Es muy importante para la administración y para la toma de decisiones, es detectar las futuras situaciones para predecir el movimiento económico y poder aplicar los correctivos necesarios. El estudio financiero es la capacidad que tiene la empresa para su liquidez, solvencia, eficiencia operativa, endeudamiento y el rendimiento y la rentabilidad, para estos análisis es necesario la utilización de ratios que son los primeros en brindar la información necesaria por medio de sus predicciones de éxito o de fracaso, para una adecuada aplicación es necesario conocer muy al detalle las actividades de la organización basándose loa ratios de aplicación pasados, el estudio financiero debe de ser aplicado por todas las empresa sin importar su ámbito de operación (Vidal-Beltrán, Erazo-Álvarez \& Narváez-Zurita, 2019).

\section{El plan de negocios como alternativa de comercialización.}

Cuando se ha fichado una oportunidad de negocios y se decide explorar, es muy fácil y sencillo empezar la creación de una empresa, al inicio la idea no parece estar ben definida, por eso que se debe de trabajarla según el producto y servicio y definiendo al segmento de mercado que se desea atender, los proveedores, establecer quienes serán los competidores y la capacidad tecnológica que se obtendrá, en esta sección el empresario ya empiece a delinear a la empresa y adjunte toda su experiencia, 


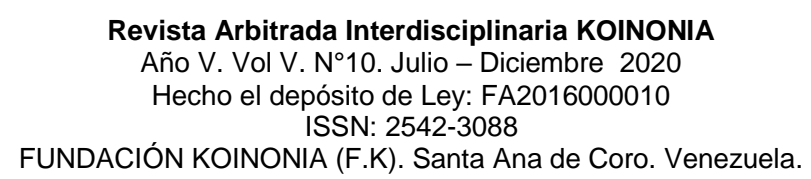

Sergio Oswaldo Zhingre Orellana; Juan Carlos Erazo Álvarez; Cecilia Ivonne Narváez Zurita; Verónica Paulina Moreno

capacidad, valores, competencias, esta idea debe de ser sólida y tener proyección es decir que debe de permanecer en el tiempo (Weinberger-Villarán, 2009).

Un plan de negocios es, un escrito claro y conciso, que anticipadamente se realizó una planeación, dicho escrito muestra los objetivos y las acciones a realizarse para la consecución de los mismos (Andía-Valencia \& Paucara-Pinto, 2014).

Un modelo de negocios es demostrar el presente y el futuro de la empresa, demostrando acciones o procesos futuros aplicando las posibles estrategias. Navarro (2015) fundamenta. "Se distinguen según las variables presente/futuro y estático/dinámico: por un lado, la descripción de una situación estática presente o inmediata de la compañía y del mercado; por el otro, la descripción del proceso futuro de desarrollo de esa situación inicial" (p.197). En el transcurso que lleva ciertas actividades para dar inicio de actividades existen algunos inconvenientes. Según (Correa-García, Ramírez-Bedoya \& Castaño-Ríos, 2010), afirman "Variables de tipo técnico, administrativo, legal, financiero y económico, por citar algunas, constituyen el punto de partida para la creación de empresas sostenibles en el tiempo" (p.181).

Este estudio nos muestra anticipadamente varios escenarios y detallar las acciones comerciales en la que se va a contribuir por lo general se presenta la estructura de la empresa y demostrar que existe un mercado con un servicio inexistente. Navarro (2015) Sostiene. "describir la situación presente/potencial proporciona una representación relevante de las variables contextuales y de la organización existente o potencial de la compañía" (p.197)

Un modelo de negocios en concreto es un conjunto de recursos con un orden cronológico, y al mismo tiempo gestionar un nuevo proyecto en un tiempo determinado en busca de convencer la factibilidad del mismo hacia los socios o lectores del plan de negocios dando a conocer los beneficios o réditos económicos. (Weinberger-Villarán, 2009), refiere que los planes de negocios se utilizan para determinar algunos casos de negocio, brindando la información necesaria a los posibles accionistas, además dando una idea de cómo funcionaría la empresa en el caso de inicio de actividades y de las actividades a realizarse, sin embargo hay algunos tipos o modelos de negocios para las 
Sergio Oswaldo Zhingre Orellana; Juan Carlos Erazo Álvarez; Cecilia Ivonne Narváez Zurita; Verónica Paulina Moreno

diferentes actividades o necesidades de cada empresa a continuación se presente algunos de los planes de negocios:

1. Planes de negocio para empresas en marcha

2. Plan de negocios para empresas nuevas

3. Plan de negocios para inversionistas

4. Plan de negocios para administradores

Para que un plan de negocio funcione correctamente se debe de tener una guía para administradores o dueños, para dirigirles en la dirección correcta teniendo la opción de realizar cambios o ajustes. Para eso a continuación se presenta los elementos necesarios que debe de tener un modelo de negocios:

1. Resumen ejecutivo: Describir los aspectos más importantes del proyecto o nuevo negocio, el receptor es lo primero que se fija para su aprobación o convencimiento.

2. Describir a la empresa o sector de negocios: Es describir a la empresa y a que productos ofrece.

3. Estado de la industria: Demostrar cómo está la empresa generalmente y sumar a esto las oportunidades que ofrece el mercado.

4. Investigación al cliente: En este sector de hace referencia al nicho de mercado que se va a satisfacer, teniendo en cuenta ingresos, cultura, etc.

5. Investigación de la competencia: Establecer cuáles son nuestros competidores directos e indirectos en el mercado seleccionado.

6. Estrategias de mercado: establecer las estrategias de las tres ( $p)$, precio, plaza y promoción y estableciendo los canales de comunicación a utilizar.

7. Operatividad: puntualizar las funciones de cada departamento de la empresa.

8. Conjunto management: se refiere a establecer un organigrama de obligaciones y responsabilidades.

9. Aplicación financiera: exponer los movimientos financieros a corto, mediano y largo plazo. 


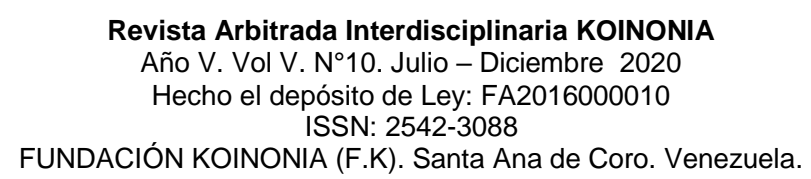

Sergio Oswaldo Zhingre Orellana; Juan Carlos Erazo Álvarez; Cecilia Ivonne Narváez Zurita; Verónica Paulina Moreno

10. Apéndice o anexos: incorporar todas las investigaciones anteriores (Balanzátegui \& Sánchez, 2017).

En un plan de negocios tambien se debe de realizar un análisis FODA que es una herramienta que diagnostica a la empresa en un entorno interno y externo, donde se estudian las fortalezas, debilidades, oportunidades y debilidades, para determinar las estrategias organizacionales micro y macro, para asi aprovechar las debilidades y oportunidades de la organización mejorando el desarrollo y su rentabilidad, incorporando la mexcla de las conocidas $4 \mathrm{P}$, llamada tambien mercadotecnia es decir la oferta completa de la empresa a los consumidores. El producto; conocido como el bien físico o intangible los que sonn expuestos a los consumidores, precio; siendo este el valor monetario de trasferencia de los tangibles o itangibles, plaza; es todo el proceso logistico que se realizar para que el bien o el servicio llegue a manos del consumidor, promocion; es una serie de actividades que se realizan para dar a conocer el producto en el mercado (Del-Rosario-Alvarado \& Cueva-Gutierrez, 2019).

En plan de negocios es un documento que se lo guarda restringidamente, los documentos originales reposan estrictamente confidencial. Navarro (2015) afirma que "Los contenidos de un Planes de Negocios muchas veces contienen información sensible sobre compañías y mercados, junto con ideas de negocios rentables e innovadoras" (p.193). Es decir el conocimiento del mismo es muy limitada y se entrega solo a personas cercanas al proyecto o el nuevo negocio, en el caso de revisarlo una persona extraña se le hace firmar un contrato de confidencialidad.

\section{MÉTODO}

La presente investigación es no experimental pues no se manipulan las variables, se analiza la situación actual de las necesidades de mercado para examinarlos y expresarlos, se utilizó un enfoque de investigación de carácter mixto, cualitativo y cuantitativo, ambos son muy valiosos y han servido para dar notables aportaciones al avance del conocimiento (Hernández, Fernandez, \& Baptista, 2014). Aun así los dos enfoques cualitativo y cuantitativo comparten estrategias generales, pero cada uno tiene 
Sergio Oswaldo Zhingre Orellana; Juan Carlos Erazo Álvarez; Cecilia Ivonne Narváez Zurita; Verónica Paulina Moreno

sus propias especialidades, pasando a la recolección de datos por medio de las encuestas que se realizó a los propietarios de vehículos.

Estos están enlazados por la evidencia de hechos, es decir mediante la tabulación de datos se obtiene indicadores que son elementos de gran importancia para nuestro estudio. Tiene un alcance descriptivo- explicativo; lo cual se describe las variables de factibilidad y plan de negocios, situaciones y eventos de los mismos explicando por qué ocurren y en qué condiciones, en este estudio se analizó las variables recopiladas en un periodo de tiempo por lo que se enfatizó una investigación transversal.

Para este desarrollo se utilizó un método histórico-lógico, con el objetivo de realizar un marco teórico y fundamentar la investigación, incluyendo el método analítico-sintético, el cual lo separa por partes: por un lado obteniendo los resultados de las encuestas y por el otro el análisis para la toma de decisiones, en este sentido, Rodríguez-Jiménez \& Pérez-Jacinto (2017), afirman que "El análisis es un procedimiento lógico y por otro lado que posibilita descomponer mentalmente un todo en sus partes y cualidades, en sus múltiples relaciones, propiedades y componentes" ( $p .8)$.

Se utilizó el método inductivo-deductivo, el inductivo; registrando y analizando los datos obtenidos y el deductivo obteniendo resultados generales de la investigación. Utilizando un enfoque sistémico que reúne varios de los elementos a una nueva totalidad, identificando la falta de proyectos de factibilidad para el sector automotriz para así poderles insinuar una solución mediante un plan de negocios. El enfoque sistémico es un acercamiento a la situación con la realidad tomándola como un todo, integrando los elementos, las relaciones y el entorno en las que se encuentran, donde escogeremos un problema y lo analizaremos a su más mínima expresión, tratando de resolverlo por separado para luego consolidar todos los elementos y tener una solución de un todo (Hernández-Altamirano \& Paredes-Gavilanez, 2018).

\section{Universo de estudio y tratamiento muestral}

El universo de estudio fue de 154.696 vehículos matriculados en la provincia del Azuay, según datos aportados por el Instituto Nacional de Estadistica y Censos (INEC, 2017). 
Sergio Oswaldo Zhingre Orellana; Juan Carlos Erazo Álvarez; Cecilia Ivonne Narváez Zurita; Verónica Paulina Moreno

En el cantón Gualaceo se registran 12.500 vehículos matriculados anualmente (EI Tiempo, 2019). De esta cantidad de vehículos se aplicó la muestra con universo finito, obteniendo 664 como resultado para nuestro objeto de estudio.

\section{Tratamiento estadístico de la información}

El tratamiento estadístico fue realizado mediante la estadística descriptiva, lo cual permitió caracterizar la información recopilada.

\section{RESULTADOS}

Para iniciar una actividad económica en un negocio esta involucra a numerosas personas como clientes, administradores, proveedores, competencia. En el sentido de la creación de un empresa comercializadora de partes y repuestos de vehiculos aportaria al desarrollo de economico y al beneficio por parte de lo propietarios de los vehiculos de la ciudad de Gualaceo. Puesto que este negocio hace contacto directo con las personas poseedoras de vehiculos, por cuanto seria un una empresa directa de distribución de partes y repuestos.

Un plan de negocios es nesesario para la consecución de este obetivo que se distinge por ser una herramienta de gran utilidada para los administradores, emprendedores, este plan nos ayuda a ver de una manera anticipada las fases especificas para que la idea de negocio plasme como un factor de éxito, este plan nos ayuda a la consecucion de los objetivos a corto, mediano y largo plazo, este plan tiene los siguientes etapas. 
Revista Arbitrada Interdisciplinaria KOINONIA

Año V. Vol V. №10. Julio - Diciembre 2020

Hecho el depósito de Ley: FA2016000010

ISSN: 2542-3088

FUNDACIÓN KOINONIA (F.K). Santa Ana de Coro. Venezuela.

Sergio Oswaldo Zhingre Orellana; Juan Carlos Erazo Álvarez; Cecilia Ivonne Narváez Zurita; Verónica Paulina Moreno

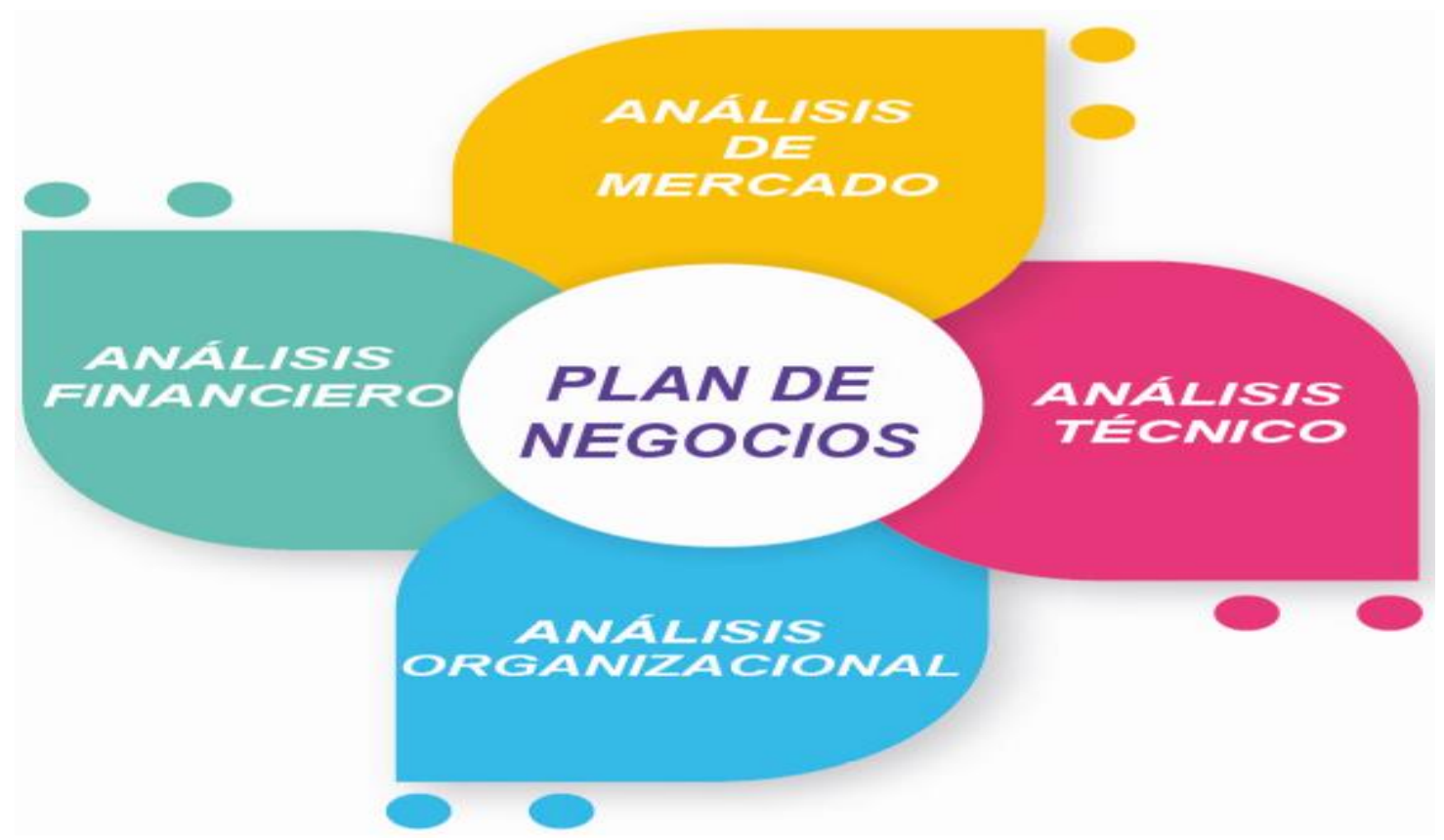

Figura 1. Estructura de un plan de negocios.

\section{Análisis de mercado}

La identificación de principales marcas de vehículos se presenta en la Figura 2

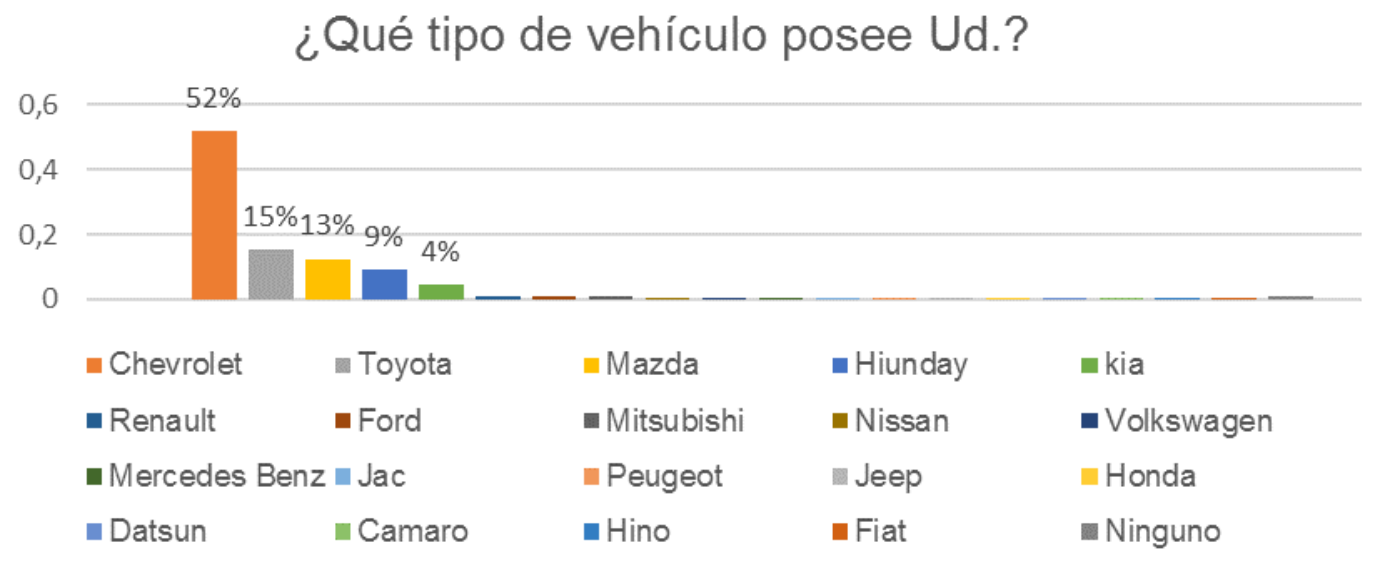

Figura 2. Identificación de las principales marcas de vehículos en la cuidad de Gualaceo. 
Sergio Oswaldo Zhingre Orellana; Juan Carlos Erazo Álvarez; Cecilia Ivonne Narváez Zurita; Verónica Paulina Moreno

Existen 20 marcas de vehículos en la cuidad de Gualaceo, liderando la marca Chevrolet con 52\% vehículos, seguido por Toyota con 15\% y Mazda con 13 .

\section{Delimitación geográfica}

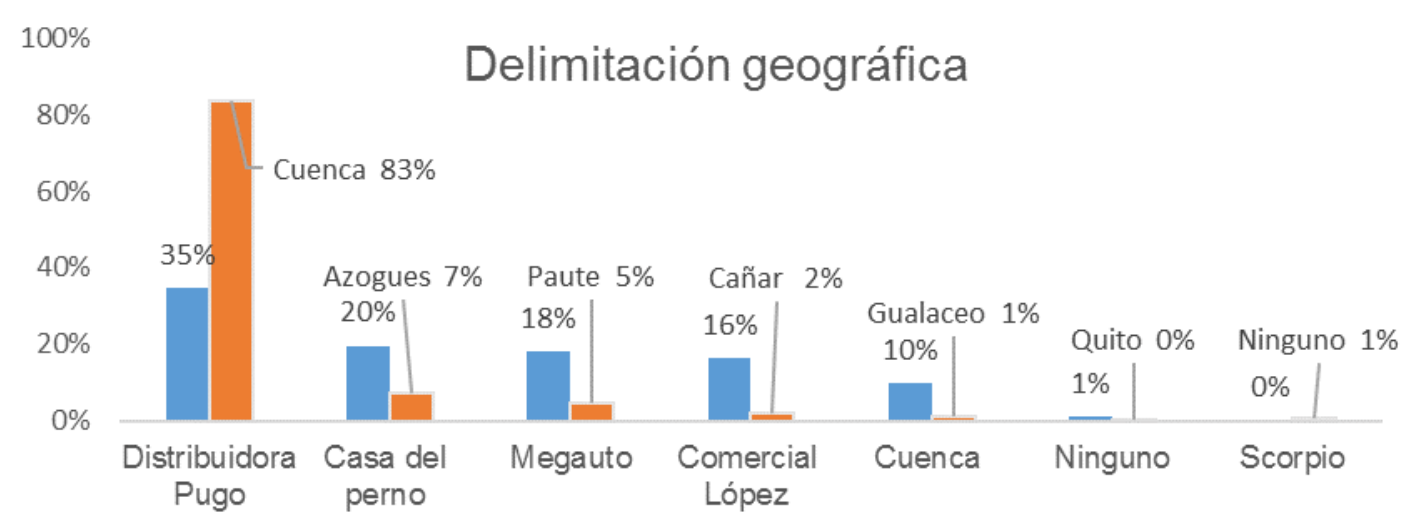

Figura 3. Delimitación geográfica de los principales almacenes de Gualaceo y a que ciudades acuden más a su compra.

En la figura 3 se analizaron los virtuales competidores, encontrando en distribuidora Pugo con el $35 \%$ el principal competidor en el cantón, Casa del perno con $20 \%$ y Megauto con 18\%, también manifestaron que al momento que no encuentran el repuesto de su necesidad, se dirigen a otras ciudades como Cuenca con el $83 \%$ por su amplia oferta y economía. 
Revista Arbitrada Interdisciplinaria KOINONIA

Año V. Vol V. N¹0. Julio - Diciembre 2020

Hecho el depósito de Ley: FA2016000010

ISSN: 2542-3088

FUNDACIÓN KOINONIA (F.K). Santa Ana de Coro. Venezuela.

Sergio Oswaldo Zhingre Orellana; Juan Carlos Erazo Álvarez; Cecilia Ivonne Narváez Zurita; Verónica Paulina Moreno

\section{Satisfacción al cliente}

\section{Satisfacción al cliente.}

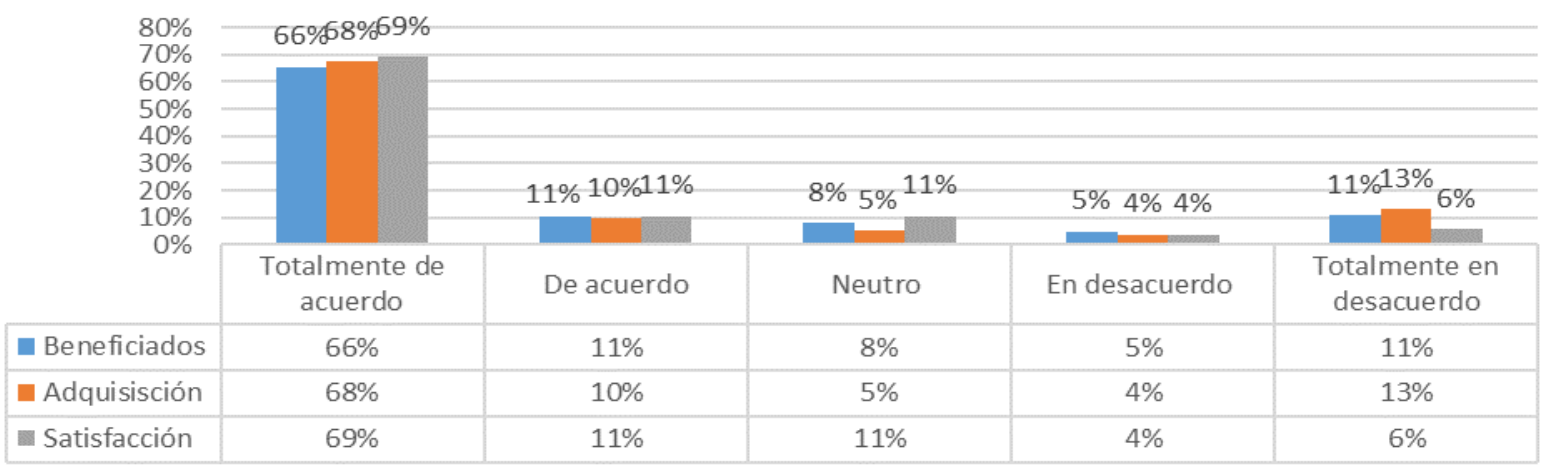

Figura 4. Satisfacción de los clientes respecto a los talleres

En términos generales existe satisfacción en las variables consultadas, $69 \%$ se sientes satisfechos con los servicios recibidos y un $68 \%$ manifiesta que existen facilidades para la adquisición. Mediante el estudio de mercado realizado, se determinó los competidores principales y la falta de inventario de acuerdo a la demanda, lo cual ocasiona inconvenientes y muchas de las veces deben movilizarse a otras ciudades principalmente a Cuenca ocasionando pérdida de tiempo y costos adicionales, también se determinaron las marcas de vehículos de más popularidad en la cuidad de Gualaceo para poder realizar los inventarios de las partes y repuestos de la marca líder que en el estudio de mercado nos reflejó la marca Chevrolet.

\section{Análisis técnico}

En este estudio se determina la dirección de la empresa que estará ubicada en la provincia del Azuay, en el cantón Gualaceo en las calles Eugenio Espejo y Antonio Delgado parte norte de la cuidad, dada la situación de apertura del local no se contratará personal por lo que este será atendido únicamente por su propietario, dispuesto a recibir el sueldo que genere el negocio. 


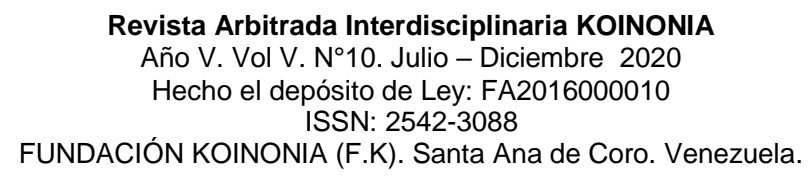

Sergio Oswaldo Zhingre Orellana; Juan Carlos Erazo Álvarez; Cecilia Ivonne Narváez Zurita; Verónica Paulina Moreno

La empresa estará dedica a la venta de repuestos genérico puesto que el ámbito de originalidad tienen exclusivamente el fabricante del vehículo, cabe decir que el repuesto genérico tiene sus propiedades como bajo costo, variedad de stock, características similares al original y durabilidad estos producidos por empresas independientes, la empresa establecerá los precios de acuerdo al promedio de mercado, a continuación se presenta mediante una tabla uno de los pedidos.

\section{Tabla 1}

Hoja de pedidos de partes y repuestos

\begin{tabular}{|c|c|c|c|c|c|}
\hline $\begin{array}{l}\text { Partes y } \\
\text { repuestos }\end{array}$ & Precio & $\begin{array}{c}\text { Línea } \\
\text { Chevrolet }\end{array}$ & $\begin{array}{c}\text { Años de } \\
\text { antigüedad }\end{array}$ & Cantidad & Total \\
\hline Faros delanteros & 45 & Chev dmax & $2015-2019$ & 4 & 180 \\
\hline Guardachoque & 60 & Chev dmax & $2015-2019$ & 4 & 240 \\
\hline Mascarilla & 35 & Chev dmax & $2015-2019$ & 4 & 140 \\
\hline Capot & 80 & $\begin{array}{l}\text { Chev dmax } \\
2015-2019\end{array}$ & $2015-2019$ & 4 & 320 \\
\hline $\begin{array}{l}\text { Guardafango } \\
\text { izquierdo }\end{array}$ & 55 & $\begin{array}{l}\text { Chev dmax } \\
2015-2019\end{array}$ & 2015-2019 & 4 & 220 \\
\hline $\begin{array}{l}\text { Guardafango } \\
\text { derecho }\end{array}$ & 55 & $\begin{array}{l}\text { Chev dmax } \\
2015-2019\end{array}$ & 2015-2019 & 2 & 110 \\
\hline Faros delanteros & 18 & Chev.luv 2.2 & $2002-2006$ & 2 & 36 \\
\hline Guardachoque & 25 & Chev.luv 2.2 & 2002-2006 & 4 & 100 \\
\hline Mascarilla & 22 & Chev.luv 2.2 & 2002-2006 & 3 & 66 \\
\hline Capot & 85 & Chev.luv 2.2 & 2002-2006 & 3 & 255 \\
\hline $\begin{array}{l}\text { Guardafango } \\
\text { izquierdo }\end{array}$ & 35 & Chev.luv 2.2 & 2002-2006 & 2 & 70 \\
\hline $\begin{array}{l}\text { Guardafango } \\
\text { derecho }\end{array}$ & 35 & Chev.luv 2.2 & 2002-2006 & 2 & 70 \\
\hline Faros delanteros & 45 & Chev.dmax & 2006-2009 & 4 & 180 \\
\hline Guardachoque & 60 & Chev.dmax & 2006-2009 & 3 & 180 \\
\hline Mascarilla & 35 & Chev.dmax & 2006-2009 & 3 & 105 \\
\hline Capot & 80 & Chev.dmax & 2006-2009 & 3 & 240 \\
\hline $\begin{array}{l}\text { Guardafango } \\
\text { Izquierdo }\end{array}$ & 55 & Chev.dmax & 2006-2009 & 3 & 165 \\
\hline $\begin{array}{l}\text { Guardafango } \\
\text { derecho }\end{array}$ & 55 & Chev.dmax & 2006-2009 & 3 & 165 \\
\hline Faros delanteros & 30 & Grand vitara & $2000-2019$ & 4 & 120 \\
\hline
\end{tabular}


Revista Arbitrada Interdisciplinaria KOINONIA

Año V. Vol V. N¹0. Julio - Diciembre 2020

Hecho el depósito de Ley: FA2016000010

ISSN: 2542-3088

FUNDACIÓN KOINONIA (F.K). Santa Ana de Coro. Venezuela.

Sergio Oswaldo Zhingre Orellana; Juan Carlos Erazo Álvarez; Cecilia Ivonne Narváez Zurita; Verónica Paulina Moreno

\begin{tabular}{llllll} 
Guardachoque & 35 & Grand vitara & $2000-2019$ & 4 & 140 \\
Mascarilla & 25 & Grand vitara & $2000-2019$ & 4 & 100 \\
$\begin{array}{l}\text { Capot } \\
\text { guardafango }\end{array}$ & 75 & Grand vitara & $2000-2019$ & 3 & 225 \\
$\begin{array}{l}\text { izquierdo } \\
\text { guardafango }\end{array}$ & 35 & Grand vitara & $2000-2019$ & 3 & 105 \\
$\begin{array}{l}\text { derecho } \\
\text { faros delanteros }\end{array}$ & 35 & Grand vitara & $2000-2019$ & 3 & 105 \\
guardachoque & 35 & Aveo activo & $2003-2019$ & 4 & 140 \\
& 30 & Aveo activo & $2003-2019$ & 4 & 120 \\
\hline
\end{tabular}

Nota: De acuerdo al estudio de mercado se trata de cubrir primero con la marca Chevrolet.

Cabe mencionar que el margen de ganancia es de $35 \%$ por unidad para recuperar la inversión inicial sobre el valor del costo de la venta para esto de analizar al proveedor que nos deje al menos precio posible tratando de que nos deje del precio promedio al menos un $15 \%$ de descuento. Respecto al análisis legal a continuación se muestra un listado de los permisos necesarios.

1. Permiso de funcionamiento del cuerpo de bomberos.

2. Patentes y permisos del lustre municipalidad de Gualaceo.

3. Se realizara la apertura del registro único de contribuyentes.

4. Inscripción en el IESS.

\section{Análisis organizacional}

Para realizar este análisis organizacional se utilizó una herramienta que incluye Fortalezas, Oportunidades, Debilidades, Amenazas (FODA), que nos permite realizar un diagnóstico situacional, tomando en cuenta los factores internos y externos de la empresa así para poder determinar nuestra situación actual. A continuación, se muestra el análisis FODA en la figura 5. 
Revista Arbitrada Interdisciplinaria KOINONIA

Año V. Vol V. №10. Julio - Diciembre 2020

Hecho el depósito de Ley: FA2016000010

ISSN: 2542-3088

FUNDACIÓN KOINONIA (F.K). Santa Ana de Coro. Venezuela.

Sergio Oswaldo Zhingre Orellana; Juan Carlos Erazo Álvarez; Cecilia Ivonne Narváez Zurita; Verónica Paulina Moreno

\begin{tabular}{|c|c|c|c|c|c|c|c|c|c|c|}
\hline \multirow{2}{*}{\multicolumn{2}{|c|}{$\begin{array}{c}\text { Mat riz FO - FA - DO - DA } \\
\text { Calificación: } 1=\mathrm{Si} / 0=\text { No } \\
\text { - ¿Mi fortaleza facilita el aprovechamiento de } \\
\text { la oportunidad? } \\
\text { - ¿Mi fortaleza facilita la superación de la } \\
\text { amenaza? } \\
\text { - ¿Mi debilidad dificulta el aprovechamiento } \\
\text { de la oportunidad? }\end{array}$}} & \multicolumn{5}{|c|}{ Oportunidades } & \multicolumn{2}{|c|}{ Amenazas } & \multirow{2}{*}{$\begin{array}{c}\text { Total } \\
\\
\text { FO FA \& } \\
\text { DO DA }\end{array}$} & \multirow{3}{*}{$\begin{array}{c}\text { ORDEN DE } \\
\text { IMPORTANCI } \\
\text { A/10 }\end{array}$} \\
\hline & & $\begin{array}{c}\text { No existe } \\
\text { almacenes que } \\
\text { se dediquen } \\
\text { exclusivamente } \\
\text { a la distribuciór } \\
\text { de partes y } \\
\text { repuestos. }\end{array}$ & \begin{tabular}{|} 
Expansión por \\
medio de los \\
talleres \\
dedicados a \\
reparaciones \\
de carrocerías \\
de vehículos.
\end{tabular} & \begin{tabular}{|c|} 
Mercado \\
insatisfecho no \\
existe los \\
productos \\
deseados
\end{tabular} & $\begin{array}{l}\text { inversión en } \\
\text { mercadería } \\
\text { diversificada }\end{array}$ & $\begin{array}{l}\text { En el mercado } \\
\text { no existe una } \\
\text { cultura de } \\
\text { trabajo en } \\
\text { función de la } \\
\text { satisfacción } \\
\text { del cliente. }\end{array}$ & $\begin{array}{l}\text { Los cambios } \\
\text { polticos, } \\
\text { económicos y } \\
\text { financieros } \\
\text { externos, por } \\
\text { parte del } \\
\text { gobierno }\end{array}$ & $\begin{array}{c}\text { Competencia } \\
\text { posesionada } \\
\text { en el mercado ( } \\
\text { pedidos a } \\
\text { Cuenca) }\end{array}$ & & \\
\hline - ¿Mi deb & $\begin{array}{l}\text { d dificulta la superación de la } \\
\text { amenaza? }\end{array}$ & 1 & 2 & 3 & 4 & 5 & 1 & 2 & 3 & \\
\hline \multirow{8}{*}{ 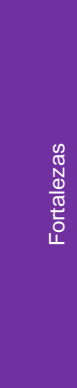 } & Local propio. & 0 & 0 & 0 & 0 & 0 & 0 & 0 & 0 & 10 \\
\hline & Líneas exclusivas de repuestos & 1 & 1 & 1 & 1 & 1 & 0 & 0 & 5 & 8 \\
\hline & $\begin{array}{l}\text { Agilidad en la entrega de } \\
\text { repuestos. }\end{array}$ & 1 & 1 & 1 & 1 & 1 & 0 & 0 & 5 & 9 \\
\hline & Competividad en precios. & 0 & 0 & 0 & 0 & 0 & 1 & 1 & 2 & 9 \\
\hline & Variedad de Productos. & 1 & 1 & 1 & 1 & 1 & 0 & 0 & 5 & 7 \\
\hline & $\begin{array}{l}\text { Segmentación de productos } \\
\text { definidos para mejorar la } \\
\text { eficiencia. }\end{array}$ & 1 & 0 & 1 & 1 & 1 & 0 & 0 & 4 & 8 \\
\hline & $\begin{array}{c}\text { Estar en búsqueda de nuevas } \\
\text { oportunidades para el } \\
\text { mejoramiento continuo. }\end{array}$ & 0 & 0 & 1 & 1 & 1 & 0 & 1 & 4 & 7 \\
\hline & Capital propio. & 0 & 0 & 0 & 0 & 0 & 1 & 0 & 1 & 10 \\
\hline \multirow{4}{*}{ 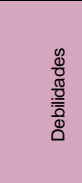 } & $\begin{array}{l}\text { Incapacidad para resolver } \\
\text { problemas inmediatos. }\end{array}$ & 0 & 0 & 0 & 0 & 0 & 0 & 0 & 0 & 7 \\
\hline & $\begin{array}{c}\text { Carencia de estudio para ofrecer } \\
\text { crédito } \\
\end{array}$ & 0 & 0 & 0 & 1 & 1 & 1 & 0 & 3 & 10 \\
\hline & Falta de un plan de marketing. & 0 & 1 & 1 & 1 & 1 & 0 & 0 & 4 & 9 \\
\hline & Uso de sistemas computarizados & 0 & 0 & 0 & 1 & 1 & 0 & 1 & 3 & 8 \\
\hline
\end{tabular}

Figura 5. Análisis y ponderación de un análisis FODA de la situación actual en la que da inicio de actividades la empresa.

Es necesario mencionar que este análisis organizacional se va a establecer según las necesidades del cliente, pues si es necesario realizar las adecuaciones necesarias se harían mediante el progreso del negocio. Dentro del direccionamiento estratégico, además se establece un nombre comercial como "Partes y repuestos Gualaceo"

Somos una micro empresa comercializadora

de partes y repuestos con productos genéricos de buena calidad hacia nuestros clientes

brindándoles una mejor opción de compra en el cantón

MISIÓN

Figura 6. Misión 
Sergio Oswaldo Zhingre Orellana; Juan Carlos Erazo Álvarez; Cecilia Ivonne Narváez Zurita; Verónica Paulina Moreno

Llegar a ser una de las comercializadoras más reconocidas del mercado local, en el sector automotriz área de partes y repuestos, caminando de la mano con la tecnología y procesos que den una mejora continua a la empresa para así brindar las comodidades necesarias a nuestros clientes

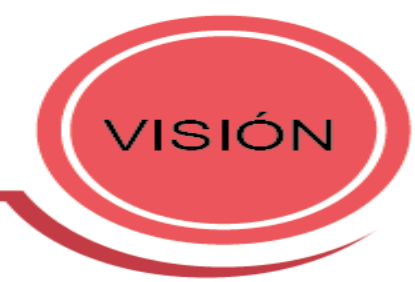

Figura 7. Visión

Se plantea dentro de análisis organizacional, estrategias del marketing mix.

Producto: Los productos a comercializar serán de marca genérica de excelente calidad y durabilidad, así también realizando el seguimiento para tener la mercadería diversificada y disponible para el cliente, además se creó un logotipo para que nuestros clientes reconozcan nuestra marca a continuación en la siguiente figura.

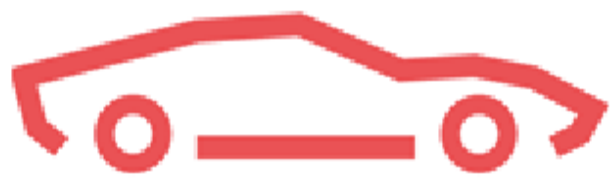

\section{Partes y repuestos Gualaceo}

Figura 8. Logotipo de la empresa para ser identificados a nivel local.

Precio: Se tratará de negociar con los proveedores para que nos realicen los descuentos al máximo para poder ingresar al mercado con una competencia en precio para así atraer a los clientes.

Plaza: La comercialización de los productos se realizará de forma local, se aplicará la venta de forma directa al consumidor y también por los medios electrónicos para el pedido de algún repuesto deseado.

Promoción: La forma de comercializar estos productos será a través de visitas a los talleres de reparaciones para dar a conocer que disponemos de los repuestos que ellos deseen y de forma directa a los clientes que se acerquen al nuestro local. 
Revista Arbitrada Interdisciplinaria KOINONIA

Año V. Vol V. N¹0. Julio - Diciembre 2020

Hecho el depósito de Ley: FA2016000010

ISSN: 2542-3088

FUNDACIÓN KOINONIA (F.K). Santa Ana de Coro. Venezuela.

Sergio Oswaldo Zhingre Orellana; Juan Carlos Erazo Álvarez; Cecilia Ivonne Narváez Zurita; Verónica Paulina Moreno

\section{Análisis financiero.}

Para el inicio de actividades es necesario estructurar un estado de situación inicial de la empresa agregando los activos corrientes, fijos y capital en este caso se cuenta con recursos propios por lo que no se tiene pasivos corrientes ni a largo plazo, cuando el negocio este en marcha si es necesario realizar un préstamo se analizara las diferentes instituciones financieras, A continuación, se presenta el estado de situación inicial.

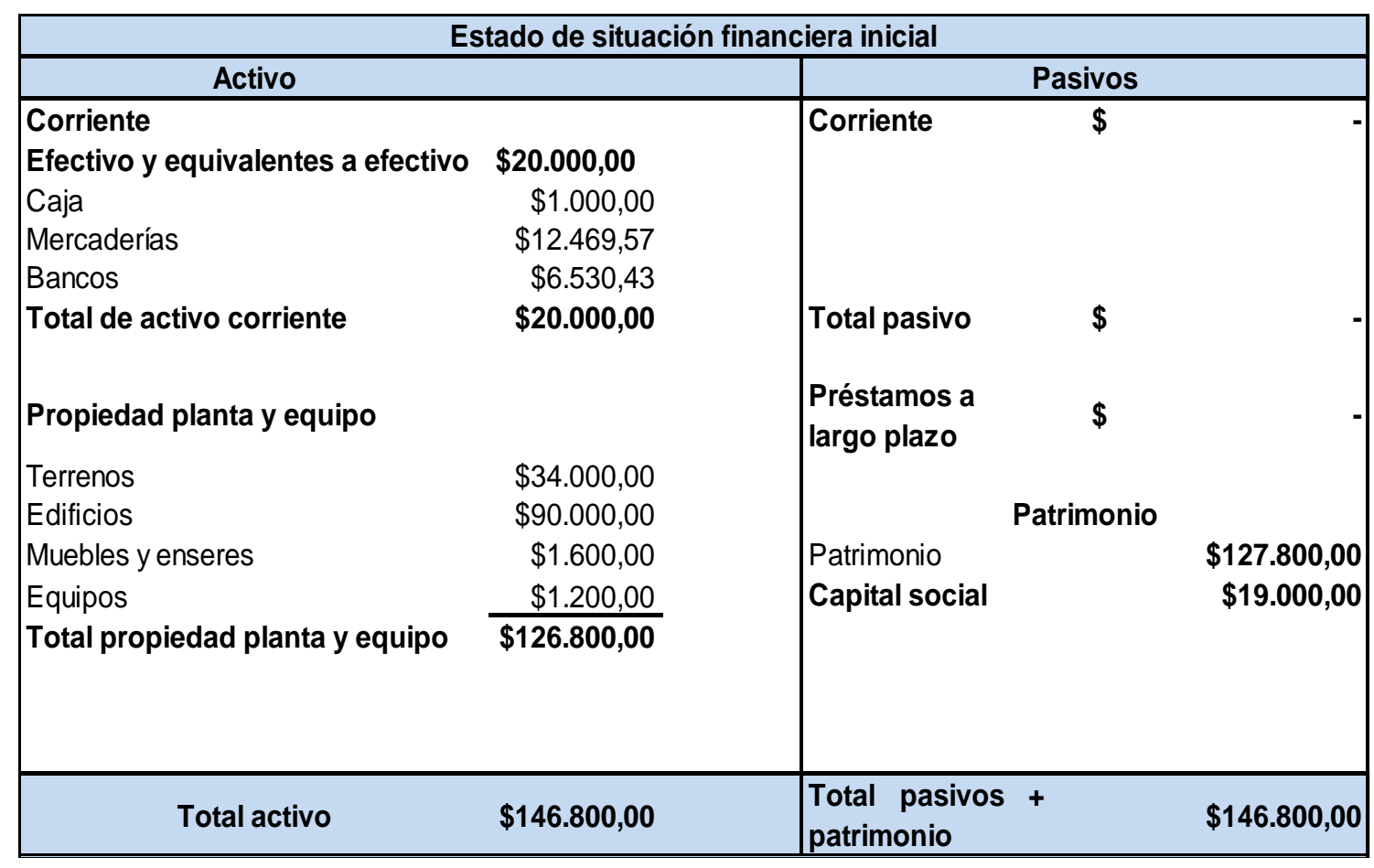

Figura 9. Estado de situación inicial antes de inicio de actividades.

Se estableció un estado de resultados proyectado a 5 años, con un crecimiento de la demanda del $10 \%$, con un incremento en los costos y gastos de una inflación de $0,023 \%$, Se presenta en la figura 10 . 
Revista Arbitrada Interdisciplinaria KOINONIA

Año V. Vol V. N¹0. Julio - Diciembre 2020

Hecho el depósito de Ley: FA2016000010

ISSN: 2542-3088

FUNDACIÓN KOINONIA (F.K). Santa Ana de Coro. Venezuela.

Sergio Oswaldo Zhingre Orellana; Juan Carlos Erazo Álvarez; Cecilia Ivonne Narváez Zurita; Verónica Paulina Moreno

\begin{tabular}{|l|l|l|l|l|l|}
\hline \multirow{2}{*}{ Concepto } & \multicolumn{5}{|c|}{ Años proyectados } \\
\cline { 2 - 6 } & \multicolumn{1}{|c|}{2021} & \multicolumn{1}{|c|}{2022} & \multicolumn{1}{|c|}{2023} & \multicolumn{1}{c|}{2024} & \multicolumn{1}{c|}{2025} \\
\hline INGRESOS & $27.732,29$ & $28.009,62$ & $28.289,71$ & $28.572,61$ & $28.858,34$ \\
Ventas & $27.732,29$ & $28.009,62$ & $28.289,71$ & $28.572,61$ & $28.858,34$ \\
Total ingresos & & & & & \\
\hline Costos & $12.469,57$ & $12.594,26$ & $12.720,20$ & $12.847,41$ & $12.975,88$ \\
\hline Mercaderías & $3.600,00$ & $3.608,28$ & $3.616,58$ & $3.624,90$ & $3.633,23$ \\
Trasporte & $1.800,00$ & $1.804,14$ & $1.808,29$ & $1.812,45$ & $1.816,62$ \\
Gastos operativos & $17.869,57$ & $18.006,68$ & $18.186,81$ & $18.284,75$ & $18.425,73$ \\
Total costos & & & & & \\
& $9.862,73$ & $10.002,94$ & $10.102,91$ & $10.287,86$ & $10.432,61$ \\
Utilidad bruta & & & & & \\
Gastos de administración & & & & & \\
Gasto administrativo & $4.800,00$ & $4.811,04$ & $4.822,11$ & $4.833,20$ & $4.844,31$ \\
Útiles de oficina & 600 & 601,38 & 602,76 & 604,15 & 605,54 \\
Servicios básicos & $1.140,00$ & $1.142,62$ & $1.145,25$ & $1.147,88$ & $1.150,52$ \\
Gastos de publicidad (Publicidad) & 600 & 601,38 & 602,76 & 604,15 & 605,54 \\
& & & & & \\
Utilidad antes de participación, & $2.722,73$ & $2.846,51$ & $2.930,03$ & $3.098,48$ & $3.226,69$ \\
utilidades & & & & & \\
Participación a empleados & 408,41 & 426,98 & 439,5 & 464,77 & 484 \\
Impuesto a la renta & 509,15 & 532,3 & 547,91 & 579,42 & 603,39 \\
\hline Utilidad neta & & & & & \\
\hline
\end{tabular}

Figura 10. Estado de resultados.

Se realizó los posibles flujos de efectivo para los primeros 5 años, agregando la inflación de $0.23 \%$ en los costos y gastos, con un aumento de participación de la demanda al $10 \%$, se puede ver la figura siguiente el flujo mencionado. 
Revista Arbitrada Interdisciplinaria KOINONIA

Año V. Vol V. Nº10. Julio - Diciembre 2020

Hecho el depósito de Ley: FA2016000010

ISSN: 2542-3088

FUNDACIÓN KOINONIA (F.K). Santa Ana de Coro. Venezuela.

Sergio Oswaldo Zhingre Orellana; Juan Carlos Erazo Álvarez; Cecilia Ivonne Narváez Zurita; Verónica Paulina Moreno

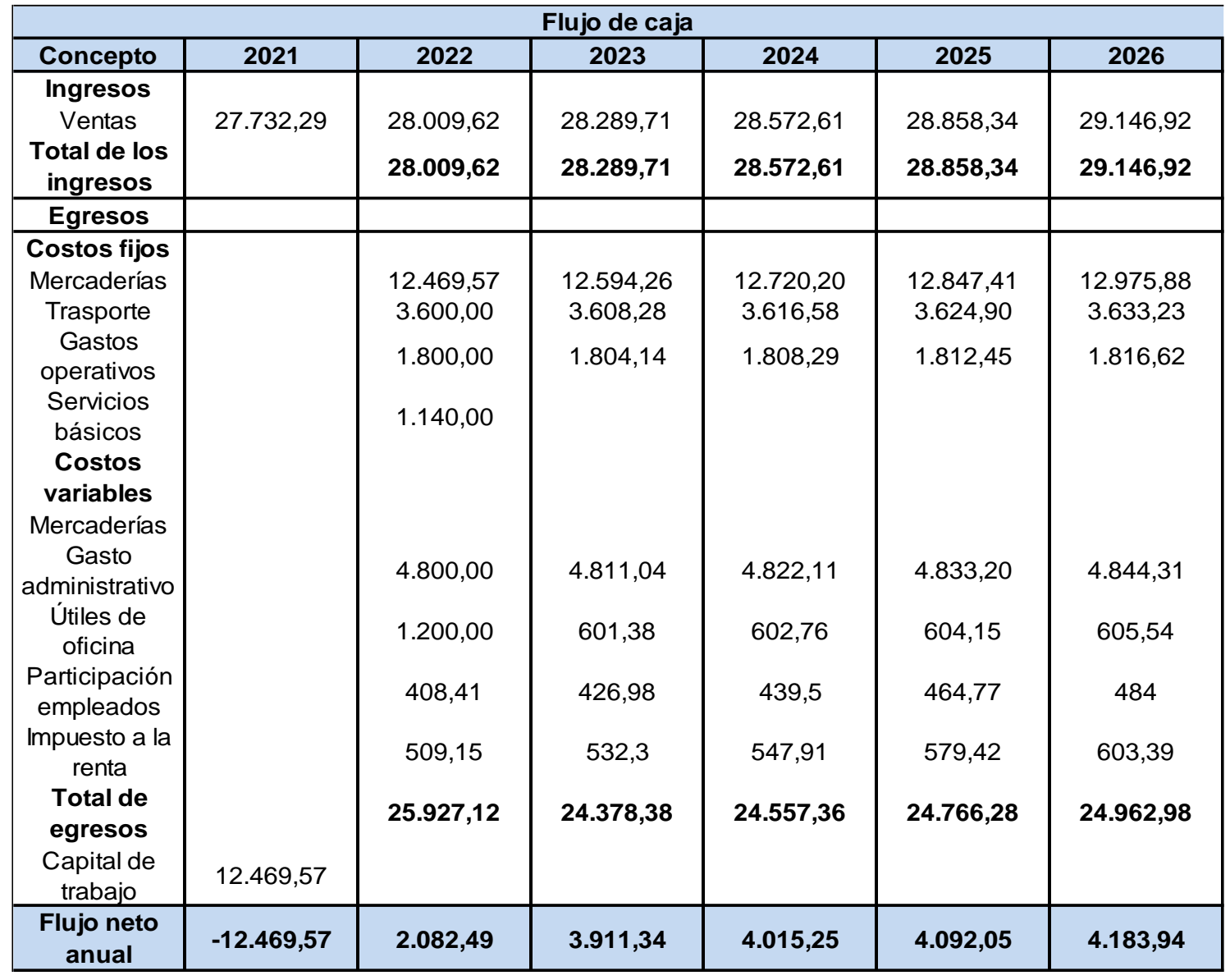

Figura 11. Flujo de caja.

\section{Valor actual neto}

Para la estimación del valor actual neto (VAN) se realizó el siguiente cálculo tomando una tasa mínima aceptable de rendimiento (TMAR) de 10\% sus cálculos fueron realizados en el programa Excel, al igual que la tasa de retorno interna (TIR), se presenta el cuadro de resumen para el cálculo de los mismos. 
Sergio Oswaldo Zhingre Orellana; Juan Carlos Erazo Álvarez; Cecilia Ivonne Narváez Zurita; Verónica Paulina Moreno

\begin{tabular}{|c|c|}
\hline Años & $\begin{array}{c}\text { Flujos de } \\
\text { caja }\end{array}$ \\
\hline 2021 & $-12.469,57$ \\
2022 & $2.082,49$ \\
2023 & $3.911,34$ \\
2024 & $4.015,25$ \\
2025 & $4.092,05$ \\
2026 & $4.183,94$ \\
\hline VAN & $24.297,86$ \\
TIR & $13 \%$ \\
\hline
\end{tabular}

Figura 12. VAN y TIR.

Para el cálculo del VAN y TIR, se utilizó las siguientes formulas.

VAN $=\frac{F 1}{(1+i)^{n}}+\frac{F 2}{(1+i)^{n}}+\frac{F 3}{(1+i)^{n}}+\frac{F 4}{(1+i)^{n}}+\frac{F 5}{(1+i)^{n}}-$ inversión inicial

TIR. Para este cálculo se iguala el VAN a cero, para ser remplazado la TMAR por la TIR.

VAN $=\frac{F 1}{(1+\text { Ktir })^{n}}+\frac{F 2}{(1+i)^{n}}+\frac{F 3}{(1+i)^{n}}+\frac{F 4}{(1+i)^{n}}+\frac{F 5}{(1+i)^{n}}-$ inversión inicial

$0=\frac{F 1}{(1+\text { Ktir })^{n}}+\frac{F 2}{(1+\text { Ktir })^{n}}+\frac{F 3}{(1+\text { Ktir })^{n}}+\frac{F 4}{(1+\text { Ktir })^{n}}+\frac{F 5}{(1+\text { Ktir })^{n}}-$ inversión inicial

Con el análisis realizado los indicadores financieros son positivos para la ejecución o inicio del negocio explicado anteriormente sabiendo que el VAN y la TIR, son netamente positivos, dando por paso o aprobación de la creación del negocio.

\section{Punto de equilibrio}

Se determina el punto de equilibrio (PE), para saber cuándo la empresa entra en un estado neutral, es decir ni le concede ni perdidas ni ganancias. Se presenta la siguiente tabla: 
Sergio Oswaldo Zhingre Orellana; Juan Carlos Erazo Álvarez; Cecilia Ivonne Narváez Zurita; Verónica Paulina Moreno

\begin{tabular}{|l|r|}
\hline \multicolumn{1}{|c|}{ Punto de equilibrio } & \multicolumn{1}{c|}{ Resultados } \\
\hline Costo fijo & $19.009,57$ \\
Costo variable & 6000,00 \\
Precio de ventas & 19359,00 \\
En unidades & 444,00 \\
En dólares & 27547,43 \\
\hline
\end{tabular}

Tabla 2. Punto de equilibrio.

\section{CONCLUSIONES}

Mediante el estudio realizado y a través de un plan de negocios se pudo constatar que en Gualaceo existe la oportunidad de negocio, de distribución de partes y repuestos gracias al crecimiento del parque automotor esto significa una oportunidad para los sabios en mercado automotriz siendo un sector muy importante para la economía local, analizando varios factores como de mercado, técnico, organizacional y financiero, demostrando así la viabilidad del negocio dando paso a la aceptación a su objetivo principal, la creación de una empresa comercializadora de repuestos.

Hoy en día toda empresa así sea pequeña, mediana o grande en la actualidad, elaborar un plan de negocio revela la información necesaria a un empresario, accionista, emprendedor analizar y conocer el mercado, es decir realizar un análisis situacional de la empresa para saber en qué circunstancias se va a competir, es necesario decir que el plan de negocios no asegura el éxito pero si nos presenta una opción mejor encaminada que nos facilitara el desarrollo del negocio para así pronosticar y advertir de los sucesos que se puedan presentar en el futuro. 
Revista Arbitrada Interdisciplinaria KOINONIA

Año V. Vol V. N¹0. Julio - Diciembre 2020

Hecho el depósito de Ley: FA2016000010

ISSN: 2542-3088

FUNDACIÓN KOINONIA (F.K). Santa Ana de Coro. Venezuela.

Sergio Oswaldo Zhingre Orellana; Juan Carlos Erazo Álvarez; Cecilia Ivonne Narváez Zurita; Verónica Paulina Moreno

\section{FINANCIAMIENTO}

No monetario.

\section{AGRADECIMIENTO}

A la Universidad Católica de Cuencas por motivar y estimular la investigación.

\section{REFRENCIAS CONSULTADAS}

Andía-Valencia, W., \& Paucara-Pinto, E. (2014). Los planes de negocios y los proyectos de inversión: similitudes y diferencias. [Business plans and investment projects: similarities and differences]. Industrial Data, 16(1), 080-084. https://doi.org/10.15381/idata.v16i1.6421

Asociación de Empresas Automotrices del Ecuador. (2018). Anuario 2018. [Yearbook 2018]. Recuperado de https://n9.cl/w30p

Argote, F., Vargas, D., \& Villada, H. (2013). Investigación de mercado sobre el grado de aceptación de mermelada de cocona en Sibundoy, Putumayo. [Market research about the acceptance level of cocona jam in Sibundoy, Putumayo]. Revista Guillermo De Ockham, 11(2), 197-206. https://doi.org/10.21500/22563202.2349

Balanzátegui, R., \& Sánchez, P. (2017). Diseño e implementación de un plan de negocios [Design and implementation of a business plan]. Observatorio de la Economía Latinoamericana. Recuperado de https://n9.cl/ig0up

Burneo -Valarezo, S., Delgado-Víctore, R., \& Vérez, M. (2016). Estudio de factibilidad en el sistema de dirección por proyectos de inversión. [Feasibility study in the project management system]. Ingeniería Industrial, XXXVII(3),305-312. Recuperado de https://n9.cl/v2ux

Correa-García, J. A., Ramírez-Bedoya, L. J., \& Castaño-Ríos, C. E. (2010). La importancia de la planeación financiera en la elaboración de los planes de negocio y su impacto en el desarrollo empresarial. [The importance of financial planning in the preparation of business plans]. Revista Facultad De Ciencias Económicas, 18(1), 179-194. https://doi.org/10.18359/rfce.2287

Del-Rosario-Alvarado, W, \& Cueva-Gutierrez, D. (2019). Plan de negocio para la integración de una línea de servicio de internet con fibra óptica para COMPUTECNICSNET. [Business plan for the integration of internet service with 
Revista Arbitrada Interdisciplinaria KOINONIA

Año V. Vol V. N¹0. Julio - Diciembre 2020

Hecho el depósito de Ley: FA2016000010

ISSN: 2542-3088

FUNDACIÓN KOINONIA (F.K). Santa Ana de Coro. Venezuela.

Sergio Oswaldo Zhingre Orellana; Juan Carlos Erazo Álvarez; Cecilia Ivonne Narváez Zurita; Verónica Paulina Moreno

optical fibre for COMPUTECNICSNET]. Revista Observatorio de la Economía Latinoamericana. Recuperado de https://n9.cl/38jt4

Dubs-de-Moya, R. (2002). El Proyecto Factible: una modalidad de investigación. [ The Feasible Project: a research modality]. Sapiens. Revista Universitaria de Investigación, 3(2); 1-18. Recuperado de https://n9.cl/yevv

El Comercio. (2019). Parque automotor de Ecuador creció en 1,4 millones de vehículos en una década. [Ecuador's automobile fleet grew by 1.4 million vehicles in a decade]. Recuperado de https://n9.cl/fnlh

El Tiempo (2019). Inicia matriculación vehicular. [Vehicle registration begins]. Recuperado de https://n9.cl/2x4q

Esparza, F., Donoso, F., Esparza, L., Parada, O., \& Janeta, A. (2017). Estudio de factibilidad para la creación de la agencia de la COAC Fernando Daquilema LTDA. [Feasibility study for the creation of the COAC Fernando Daquilema agency LTD]. Revista Observatorio de la Economía Latinoamericana, Ecuador, 231. Recuperado de https://n9.cl/ws6i

Hernández-Altamirano, H, \& Paredes-Gavilanez, J. (2018). El pensamiento sistémico en las organizaciones del Ecuador. [Systemic thinking in organizations in Ecuador]. Revista Observatorio de la Economía Latinoamericana. Recuperado de: https://n9.cl/cgq98

Hernández, R., Fernandez, C., \& Baptista, L. P. (2014). Metodologia de la investigación. [Investigation methodology]. México: McGraw-Hill.

Instituto Nacional de Estadisticas y Censos (INEC, 2017). Anuario de estadisticas de trasporte 2017. [Transportation Statistics Yearbook 2017]. Recuperado de https://n9.cl/e6eb

Instituto Nacional de Estadisticas y Censos (INEC, 2016). El parque automotor de Ecuador creció $57 \%$ en cinco años. [Ecuador's automotive fleet grew $57 \%$ in five years]. Recuperado de https://n9.cl/htrh

Marín, K., \& Atencio, E. (2008). Proceso Académico-Administrativo de la Revista Científica y Ética del Investigador: estudio de un caso. [Academic-Administrative Process of the Researcher's Scientific and Ethical Journal: a case study]. Omnia, 14(3); 160-183. Recuperado de https://n9.cl/fclp

Murillo-Vilela, C., Erazo-Álvarez, J., Quevedo-Vázquez, J., \& Narváez-Zurita, C. (2019). Plan de inversión como herramienta de la gestión del capital 
Revista Arbitrada Interdisciplinaria KOINONIA

Año V. Vol V. N¹0. Julio - Diciembre 2020

Hecho el depósito de Ley: FA2016000010

ISSN: 2542-3088

FUNDACIÓN KOINONIA (F.K). Santa Ana de Coro. Venezuela.

Sergio Oswaldo Zhingre Orellana; Juan Carlos Erazo Álvarez; Cecilia Ivonne Narváez Zurita; Verónica Paulina Moreno

intelectual. [Investment plan as a tool for intellectual capital management]. Revista Arbitrada Interdisciplinaria Koinonía, 4(1), 245-273. http://dx.doi.org/10.35381/r.k.v4i1.458

Navarro, F. (2015). Análisis situado del plan de negocios en español y portugués: perspectivas de emprendedores, docentes y estudiantes. [Situated analysis of the business plan in Spanish and Portuguese: perspectives of entrepreneurs, teachers and students]. Calidoscópio, 13(2); 189-200. doi:10.4013/cld.2015.132.05

Padilla-Campoverde, D., Erazo-Álvarez, J., \& Narváez-Zurita, C. (2019). Diagnóstico situacional del potencial turístico de la parroquia Ricaurte, Azuay. [Situational diagnosis of the tourist potential in Ricaurte parish, Azuay]. Revista Arbitrada Interdisciplinaria Koinonía, 4(1), 376-407. doi:http://dx.doi.org/10.35381/r.k.v4i1.462

Peña-Abreu, M., Rodríguez-Rodríguez, C. R., \& Piñero-Pérez, P. Y. (2017). Computación con palabras para el análisis de factibilidad de proyectos de software. [Word Computing for Software Project Feasibility Analysis]. Tecnura, 20(50), 69-84. Recuperado de https://n9.cl/sduzi

Puente, M., \& Carrasco, V. (2017). Plan de negocios una guía empresarial para pequeños negocios. [Business plan, a corporate guide for small businesses]. Revista Observatorio de la Economía Latinoamericana. Recuperado de: https://n9.cl/h2bu

Rodríguez-Jiménez, A., \& Pérez-Jacinto, A. (2017). Métodos científicos de indagación y de construcción del conocimiento. [Scientific methods of inquiry and knowledge construction]. Revista Escuela De Administración De Negocios, (82), 175-195. https://doi.org/10.21158/01208160.n82.2017.1647

Vidal-Beltrán, K., Erazo-Álvarez, J., \& Narváez-Zurita, C. (2019). La lógica difusa como herramienta de evaluación financiera de proyectos de inversión. [Fuzzy logic as a financial evaluation tool for investment projects]. Revista Arbitrada Interdisciplinaria Koinonía, 4(1), 309-348. http://dx.doi.org/10.35381/r.k.v4i1.460

Weinberger-Villarán, K. (2009). Plan de negocios. Herramienta para evaluar la viabilidad de un negocio. [Business plan. A tool to assess the viability of a business]. Recuperado de https://n9.cl/r2qea

(C2020 por los autores. Este artículo es de acceso abierto y distribuido según los términos y condiciones de la licencia Creative Commons Atribución-NoComercial-Compartirlgual 4.0 Internacional (CC BY-NC-SA 4.0)

(https://creativecommons.org/licenses/by-nc-sa/4.0/). 